\title{
High-Resolution Accurate Mass Measurements of Biomolecules Using a New Electrospray Ionization Ion Cyclotron Resonance Mass Spectrometer
}

\author{
Brian E. Winger, Steven A. Hofstadler, James E. Bruce, Harold R. Udseth, \\ and Richard D. Smith \\ Chemical Methods and Separations Group, Chemical Sciences Department, Pacific Northwest Laboratory, \\ Richland, Washington, USA.
}

\begin{abstract}
A novel electrospray ionization/Fourier transform ion cyclotron resonance mass spectrometer based on a $7-\mathrm{T}$ superconducting magnet was developed for high-resolution accurate mass measurements of large biomolecules. Ions formed at atmospheric pressure using electrospray ionization (ESI) were transmitted (through six differential pumping stages) to the trapped ion cell maintained below $10^{-9}$ torr. The increased pumping speed attainable with cryopumping ( $>10^{5} \mathrm{~L} / \mathrm{s}$ ) allowed brief pressure excursions to above $10^{-4}$ torr, with greatly enhanced trapping efficiencies and subsequent short pumpdown times, facilitating high-resolution mass measurements. A set of electromechanical shutters were also used to minimize the effect of the directed molecular beam produced by the ESI source and were open only during ion injection. Coupled with the use of the pulsed-valve gas inlet, the trapped ion cell was generally filled to the space charge limit within $100 \mathrm{~ms}$. The use of $10-25 \mathrm{~ms}$ ion injection times allowed mass spectra to be obtained from $4 \mathrm{fmol}$ of bovine insulin $\left(M_{\mathrm{r}} 5734\right)$ and ubiquitin $\left(M_{r} 8565\right)$. with resolution sufficient to easily resolve the isotopic envelopes and determine the charge states. The microheterogeneity of the glycoprotein ribonuclease $\mathbf{B}$ was examined, giving a measured mass of $14,898.74$ Da for the most abundant peak in the isotopic envelope of the normally glycosylated protein (i.e., with five mannose and two $\mathrm{N}$-acetylglucosamine residues (an error of approximately $2 \mathrm{ppm}$ ) and an average error of approximately 1 ppm for the higher glycosylated and various $\mathrm{H}_{3} \mathrm{PO}_{4}$ adducted forms of the protein. Time-domain signals lasting in excess of $80 \mathrm{~s}$ were obtained for smaller proteins, producing, for example, a mass resolution of more than 700,000 for the $4^{+}$charge state ( $m / z$ 1434) of insulin. (I Am Soc Mass Spectrom 1993, 4, 566-577)
\end{abstract}

$\mathrm{B}$ uilding on the early results of Dole et al. [1], Yamashita and Fenn [2] and Aleksandrov et al. [3] successfully combined electrospray ionization (ESI) with mass spectrometry in the mid-1980s and provided the foundation for the explosive growth into new areas of mass spectrometric application $[4,5]$. In particular, ESI mass spectrometry (ESI/MS) has opened new avenues for the study of large biopolymers. The efficient ionization process and the multiple-charging phenomenon inherent in ESI have provided high sensitivities, the combination with high-performance liquid chromatngraphy and capillary electrophoresis, and the observation of high-molecular-weight species within a limited mass-to-charge ratio observation window [5]. An advantage of ESI over other ionization methods is its flexibility, which is underscored by the fact that the

Address reprint requests to Richard D. Smith, Chemical Methods and Separations Group, Chemical Sciences Department, Pacific Northwest Laboratory, P.O. Box 999, MS P8-19, Richland, WA 99352. major types of mass spectrometric instrumentation [i.e., quadrupoles, magnetic sectors [3, 6], time-of-flight [7]. quadrupole ion traps [8], and Fourier transform ion cyclotron resonance (FT-ICR) [9]], have been successfully coupled to ESI sources.

In addition to molecular weight information, ESI/MS can provide structural information on peptides and proteins. For example, the observed gasphase charge state distribution produced by ESI is apparently related to molecular structure and solution-phase conditions $[2,10]$. Fragmentation patterns can potentially provide partial sequence information [11], even for large proteins [12], and their understanding would be greatly facilitated by more accurate determination of both mass and charge state of the products. The identification of adduct peaks can contribute information on the chemical nature of biomolecules and associations in solution. Combined contributions due to fragmentation, adduction, and molecular heterogeneity, however, can result in spec- 
tra that are extremely complicated. In fact, existing deconvolution schemes designed to aid the interpretation of electrospray data [13] provide evidence of the complex nature of such mass spectra. Without high mass resolution, at least high enough to assign unambiguously charge states to all observed ions, full use cannot be made of complex ESI mass spectra of large biopolymers. High-resolution mass spectra of proteins have been obtained for both molecular and fragment ions $\left[9_{c}, 14\right]$. With the capability of determining charge state on the basis of spacing of the isotope peaks, molecular weight determinations with accuracies in the low parts per million range have been made [14]. Of direct relevance to this work are the pioneering contributions by McLafferty and co-workers [9], who have demonstrated the potential for high-resolution ESI/MS using FT-ICR.

We present here initial results of our efforts at coupling the high-ionization efficiency of ESI with the high-resolution and accurate mass analysis capabilities intrinsic to the FT-ICR mass spectrometer [15]. With the use of six differential pumping stages, two electromechanical shutters, and a novel rryopump assembly in the final pumping stage, ions produced at atmospheric pressure were mass analyzed below $10^{-9}$ torr. In an effort to understand the trapping of electrosprayed ions, we investigated ion kinetic energies and kinetic energy distributions produced by the ESI source and how these parameters relate to optimal trapping conditions. Mass spectra are presented, with resolu- tions extending to more than 700,000 for small proteins. We also demonstrate the application to more complex mixtures, with an average mass accuracy of approximately 1 ppm for higher glycosylated and adducted forms of ribonuclease (RNase) B, as well as the potential for simultaneously obtaining both high resolution and high sensitivity.

\section{Experimental}

\section{ESI/FT-ICR Instrumentation}

The novel ESI/FT-ICR mass spectrometer described herein consisted of a modified Analytica (Branford, CT) ESI source interfaced to a modified IonSpec external source FT-ICR (IonSpec, Irvine, CA). The entire system, illustrated in Figure 1, consists of six differentially pumped vacuum stages that allow sampling of the ion population formed by ESI at atmospheric pressure and subsequent mass analysis at pressures below $10^{-9}$ torr.

The ESI source was modified by replacing the glass ion-inlet capillary with a $1-\mathrm{mm}$ inner diameter (ID) $x$ 27-cm-long stainless steel capillary that was resistively heated with an Electronic Measurements Inc. (Neptune, NJ) model TCR $205501000-W$ power supply. To avoid heat deformation of source components due to the higher temperature operation, all Delrin insulators were replaced with Vespel components. The first vac uum stage (i.e., the capillary-skimmer interface) was
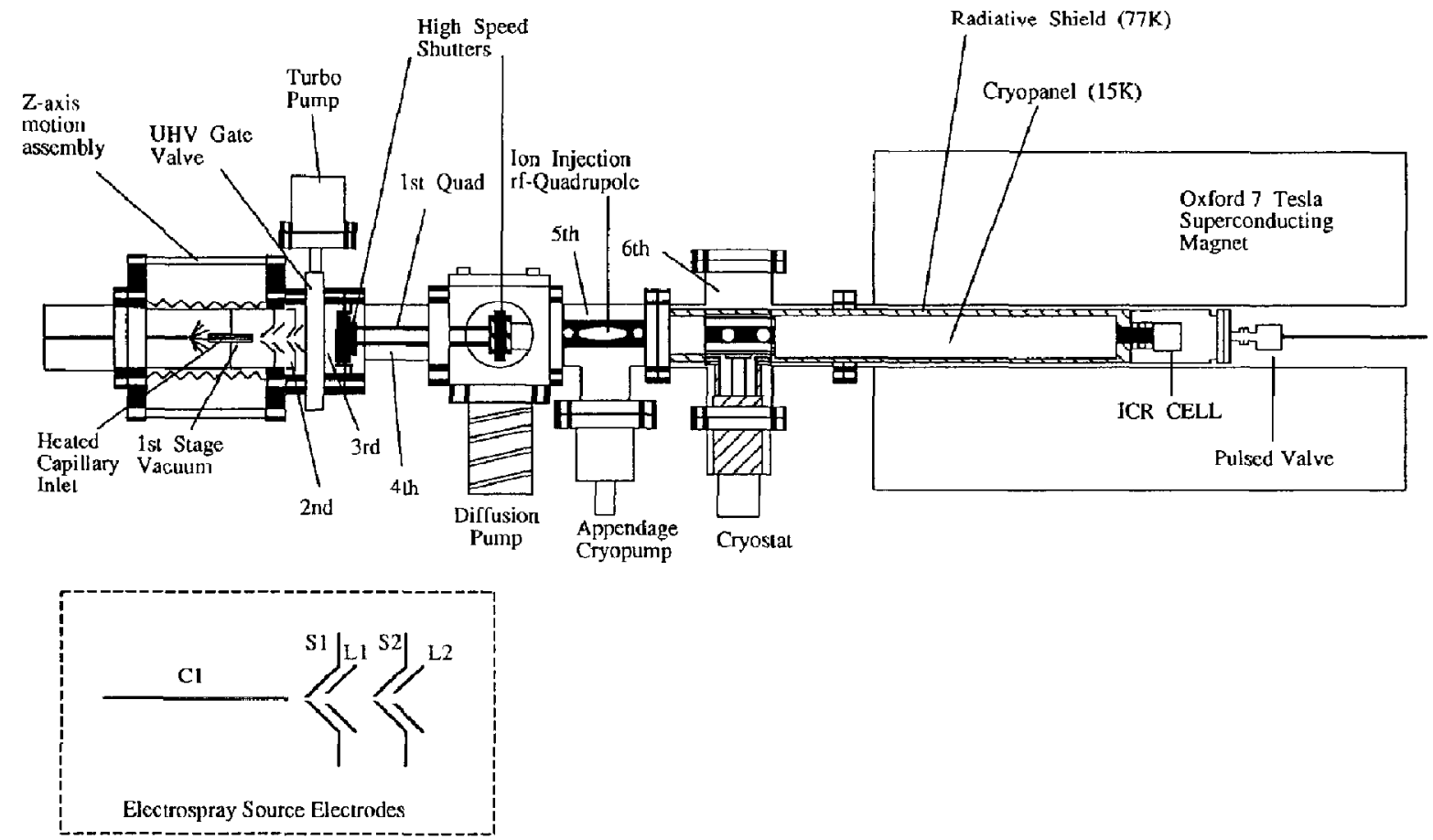

Figure 1. Schematic representation of the FT-ICR instrumentation used to acquire high resolution and accurate mass measurements of electrosprayed biomolecules; inset, ESI source interface region. 
maintained at a pressure of $3.5-4.0$ torr by using a $667-\mathrm{L} / \mathrm{min}$ mechanical rotary pump. The second stage was separated from the first stage with a $1.5-\mathrm{mm}-\mathrm{ID}$ skimmer located $4.5 \mathrm{~mm}$ from the exit of the capillary, upstream of the Mach disk. The second stage, which was separated from the third stage by using a $2.5-\mathrm{mm}$ ID skimmer, was evacuated to a pressure of $90 \mathrm{mtorr}$ by using a $230-\mathrm{L} / \mathrm{s}$ roots blower backed with a 667 $\mathrm{L} /$ min mechanical rotary pump. A conical lens (L.1) was placed between the first and second skimmers to collimate better the beam [16], and a second conical lens (L2) was mounted just beyond the second skimmer. The ESI source elements are illustrated in Figure 1 (inset). The ESI source was mounted on a Thermionics (Hayward, CA) model ECT-6-3 welded bellows, producing a $z$-axis translation stage with $7.6 \mathrm{~cm}$ of $z$-axis motion. When the bellows was fully extended, the distance from the source (back of L2) to the first quadrupole entrance was approximately $3.8 \mathrm{~cm}$. Compression of the bellows allowed L2 to be moved through the center of the gate valve to within $0.03 \mathrm{~cm}$ of the quadrupole entrance. A 50- $\mathrm{L} / \mathrm{s}$ turbomolecular pump, which was mounted on the upper portion of the gate valve, was used to evacuate the third vacuum stage to approximately $10^{-4}$ torr. The fourth vacuum stage, which contained the first quadrupole segment, was evacuated to $10^{-5}$ torr with a $500-\mathrm{L} / \mathrm{s}$ diffusion pump, and the fifth stage was evacuated with a conventional $1250-\mathrm{T} . / \mathrm{s}$ cryopump. (No pressure-measuring device was placed in the fifth stage.)

Electromechanical shutters provided by IonSpec were mounted on both sides of the first quadrupole segment and were open only during injection of the ion beam into the trapped ion cell. The shutters were normally closed, blocking a $3.5-\mathrm{mm}$ orifice on each shutter plate and minimizing the time that the directed molecular beam emanating from the ESI source could traverse the differential pumping regions and adversely affect the cell pressure. The shutters were driven by a miniature vacuum-compatible solenoid with a minimum cycle time of $5 \mathrm{~ms}$. With the ESI source in operation and the electromechanical shutters in the open position for several seconds, the pressure in the trapped ion cell region would typically increase by nearly $4 \times 10^{-10}$ torr. A second 1 -m-long quadrupole segment, located between the second shutter and the trapped ion cell, was used to facilitate transfer of the ion beam through the fringing magnetic field $[17,18]$ to the cell. The final vacuum stage, which contained the trapped ion cell, was evacuated with a custom cryopump, developed in our laboratory, consisting of two dual concentric cylinders. The outer cylinder, which serves as the radiative heat shield, was made of 4.25-in, outer diameter $(O D) \times 0.063$-in.-thick aluminum. The inner cylinder, which constitutes the cryopumping surface, was made of 3.5-in. OD $\times 0.062-$ in.-thick OFHC copper. Both cylinders were mechanically polished to a $16-\mu \mathrm{m}$ finish to reduce surface emissivity. Reduction of the surface emissivity reduces the amount of radiative energy absorbed and diminishes the possibility of large temperature gradients along the length of the cylinders. The first set of cylinders was approximately $10.2 \mathrm{~cm}$ long, whereas the second set of cylinders was approximately $55.6 \mathrm{~cm}$ long and extended into the bore of the superconducting magnet. The entire assembly was mounted on an APD-8 cryostat (APD Cryogenics, Allentown, PA). The radiative shield (outer cylinder) was cooled to approximately $77 \mathrm{~K}$, and the cryopumping surface (inner cylinder) was cooled to $15 \mathrm{~K}$. A second inner radiative heat shield was not implemented owing to space limitations. Nevertheless, this pumping configuration circumvents conductance limitations that plague traditional pumping configurations and provides a pumping speed of more than $10^{5} \mathrm{~L} / \mathrm{s}$, resulting in an operating pressure in the cell below $10^{-9}$ torr. A magnetically compatible piezoelectric pulsed gas inlet (Laser Technics, Albuquerque, NM) was used to enhance ion accumulation in the cell by the introduction of gas to the cell region during ion injection. A brief pressure excursion to over $10^{-4}$ torr is achieved at the cell, with the subsequent pumpdown to base pressure typically requiring less than $5 \mathrm{~s}$.

\section{Ion Trupping and Detection}

Efficiency of ion trapping and its relationship to the overall FT-ICR performance were evaluated under a variety of experimental conditions. To determine the effect of pressure on ion trapping efficiency, a comparison was made at $10^{-6}$ torr (achieved without the use of the cryopump assembly), at a static pressure below $10^{-9}$ torr, and with pulsed gas pressure excursions to over $10^{-4}$ torr during the injection period. Trap potentials were independently optimized in the range $0.1-10$ $\mathrm{V}$ for both the ion accumulation and detection events. The ion injection sequence without the use of the piezoelectric pulsed valve involved pulsing the quadrupole radiofrequency (RF) and opening the electromechanical shulters for ion accumulation periods of 2-30 $\mathrm{s}$, depending on the sample. With the use of the pulsed piezoelectric valve, $\mathrm{N}_{2}$ gas pulses of 25-225 ms into the analyzer region were used, with ion injection times of $10-500 \mathrm{~ms}$ (during which the cell was filled to the space charge limit). Trapping voltages were chosen based on the translational energy of the ion beam, which was experimentally determined by retarding potential analysis (see Discussion). Following a variable delay of up to $60 \mathrm{~s}$ to allow for collisional cooling, the trapping voltages were lowered, typically to 250 $\mathrm{mV}$. Ions were excited to larger cyclotron orbits with a linear excitation at a sweep rate of $25 \mathrm{~Hz} / \mu$ s over the frequency range of interest. The amplitude of the excitation voltage was optimized for each protein studied.

Data acquisition was performed by using two different schemes. The majority of the data presented here was obtained by using the IonSpec Omega data system, software version 3.03. Spectra were acquired 
over a bandwidth that coincided with the excitation bandwidth. The maximum number of data points (256K) was acquired for ail spectra to maximize resolution. A separate acquisition system, consisting of an IBM 80286 AT machine, Data Translation (Marlboro, MA) Global Lab acquisition software (a menu-driven package for high-speed acquisition, display, and analysis), and a DT2821 12-bit 50-kHz digitizer, was used to acquire extended time-domain signals. Because the output of the DT2821 is written directly to the disk drive rather than to random access memory, the maximum length of the time-domain signal was limited only by the disk size ( $600 \mathrm{~s}$ for a 30-Mbyte hard drive). The longest experimentally acquired transdomain signal was $80 \mathrm{~s}$ for both bovine insulin and bovine ubiquitin. After zero filling once, the resulting signal was Fourier transformed, and a calibration equation originally generated by the Omega data system was applied to the magnitude mode frequency domain data to obtain the mass-to-charge ratio spectrum.

\section{ESI and External Source Injection}

Proteins were used as received from Sigma Chemical Co. (St. Inuis, MO). Protein solutions of $0.5 \mathrm{mg} / \mathrm{mL}$ in an aqueous $5 \%$ acetic acid were infused at $0.1-1.0$ $\mu \mathrm{L} /$ min using a Harvard Apparatus (South Natick, MA) 22 syringe pump. In addition, a $2.0-\mu \mathrm{L} / \mathrm{min}$ methanol sheath flow was infused with a second Harvard Apparatus pump and mixed at the point of electrospray [19]. ESI voltages of approximately +3.5 to $+4.5 \mathrm{kV}$ were applied for production of positive ions. The inlet capillary was heated to approximately $100150{ }^{\circ} \mathrm{C}$, with an average of $32 \mathrm{~W}$ applied. The capillary was held at +150 to $+350 \mathrm{~V}$, whereas the first skimmer potential was typically $+50 \mathrm{~V}$. This potential difference, in addition to providing capillary heating, aided ion desolvation. The second skimmer and other source lenses were held at potentials that optimized the signal intensity and minimized the energy spread. Optimal ion current was obtained at the trapped ion cell when the quadrupole entrance plates and the quadrupole offset were held at $0 \mathrm{~V}$. The overall transmission efficiency of the dual quadrupole injection system was determined by comparing the total ion current (TIC) exiting the ESI source to the TIC arriving at the trapped ion cell. With control electronics designed by IonSpec, a $2.27-\mathrm{MHz}$ signal variable from 0 to $540 \vec{V}_{p-p}$ was applied to the quadrupole rods to obtain efficient (approximately 50\%) transmission of the ion packet from the exit of the ESI source to the trapped ion cell. The TIC exiting the ESI source was measured on shutter 1 when it was closed, whereas the TIC measured at the cell was the sum of the ion current impinging on the rear trap plate and the ESI source filament (mounted directly behind the rear trap plate). Typical ion currents traversing the trapped ion cell were 10-30 $\mathrm{pA}$, depending on solution conditions and ESI source potentials. The transmission efficiency through the individual quadrupole segments was better than 90\%, whereas ion loss at the junction of the two quadrupoles was typically approximately $30 \%$.

\section{Results and Discussion}

\section{Trapping of Externally Generated Ions}

The multiple-element ESI source shown in Figure 1 (inset) allows fine control of the kinetic energy characteristics of the ion beam injected into the quadrupole ion guide. The degree of dissociation in the nozzle-skimmer region, and to some extent the degree of desolvation, can be controlled by the potential difference between the inlet capillary and the first skimmer cone [20], similar to that reported earlier for nozzle-skimmer interface designs [21]. The mean kinetic energy, as well as the kinetic energy distribution of the ion beam, was manipulated by adjusting the potentials applied to electrodes L1, S2, and L2. A typical electrostatic configuration in which $\mathrm{C} 1, \mathrm{~S} 1, \mathrm{~L} 1, \mathrm{~S} 2$, and $\mathrm{I} 2$ are biased at $+350,+51.2,+57.4,-0.3$, and $+5.8 \mathrm{~V}$. respectively, was found to yield a mean kinetic energy of $8 \mathrm{eV} / q$ (where $q$ is the ion net charge), with an average kinetic energy distribution of $3 \mathrm{eV} / q$ (fullwidth at half-maximum). (Ion energies in this work are given divided by ion charge state in units of electron volts per $q$, so as to minimize confusion with regard to electrostatic potentials.) The kinetic energy of the ion beam is defined by the velocity component achieved in the supersonic expansion plus the component due to the electric fields imposed by the lens elements [22]. In these studies, once a suitable skimmer capillary distance was found, the gas dynamics of the system was held constant, and only electrostatic manipulation was used to adjust kinetic energies. Ion kinetic energy profiles were generated by varying the offset on the first quadrupole and measuring the ion current transmitted to the second shutter [23]. The mean kinetic energy and the kinetic energy distribution of the ion beam were obtained by taking the first derivative of this profile (i.e., $\Delta I / \Delta V$, as shown in Figure 2). The resulting ion beam is in the kinetic energy regime that is most conducive to trapping (i.e., below $10 \mathrm{eV} / q$ ). The available voltage range of the trap plates $( \pm 10 \mathrm{~V})$ was modified to allow switching between two predetermined voltage levels; this allows conditions suitable for both efficient trapping and detection.

The first successful coupling of ESI with FT-ICR [9a] was performed at low pressures, utilized exclusively gated trapping, and required lengthy relaxation delays to achieve high resolution $[9 a, c]$. An alternative trapping scheme, referred to as accumulated trapping, was subsequently demonstrated to be highly compatible with the continuous nature of the ESI source [22, 24-26] but was effective at pressures significantly above those considered optimal for high-performance 


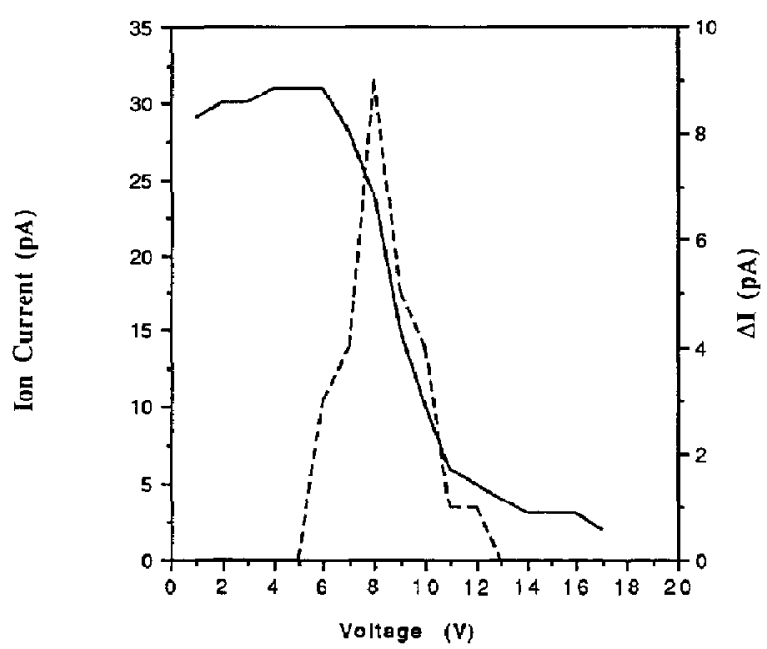

Figure 2. Ion kinetic energy profile generated by varying the offset voltage on the first quadrupole segment and measuring the ion current transmitted to the second shutter (solid curve); first derivative $\Delta I / \Delta V$ of the kinetic energy profile (dashed curoe). Ions were generated from a $0.5-\mu \mathrm{g} / \mu \mathrm{L}$ solution of horse myoglobin in $1 \%$ acetic acid.

FT-ICR. Recently, McLafferty and co-workers [9d] have demonstrated high-resolution ESI/FT-ICR mass measurements with the use of a pulsed gas inlet that allowed for both high-pressure accumulated trapping and low-pressure ion detection.

Another trapping scheme that utilizes changes in electrode potentials involves the application of voltages transverse to the $z$-axis. With this method, offset potentials are applied either to an auxiliary electrode outside the trapped ion cell [27] or directly to the excitation/detection electrodes [28]. The applied transverse potential creates a perturbation in the electric field, resulting in enhanced accumulation efficiency. One major disadvantage that this method has in common with gated trapping is that whereas ion accumulation in the cell may be enhanced, the trapped ion packet has no efficient mechanism to relax to the center of the potential well where electric fields for both trapping and excitation are more ideal [29-31]. In addition, for long ion accumulation times, the applied offset induces increases in magnetron motion, resulting in the ion packet "walking" out of the cell. Alternatively, an azimuthal quadrupolar field can be applied at the unperturbed cyclotron frequency [32], resulting in conversion of magnetron motion directly to cyclotron motion [33]. To efficiently "cool" large ions to the center of the cell for optimum excitation and detection, cooling times of several minutes $[9 a-c]$ are required at high vacuum. To reduce cooling times, the pressure in the trapped ion cell region can be increased by several orders of magnitude with the pulsed introduction of a cooling gas prior to excitation and detection.
The increased density of background neutrals increases the collision frequency for ions oscillating along the $z$-axis and results in shorter cooling times. Ion cooling along the $z$-axis occurs as neutrals carry away excess energy. This mechanism is useful both for trapping ions in a static potential well and subsequently cooling the confined ion cloud. Ions can be efficiently trapped at high pressure (i.e., $10^{-5}-10^{-7}$ torr) simply by matching the kinetic energy of the ion beam with the applied trapping potential [25].

Preliminary studies were undertaken in which the cryopumping assembly was not used, and pressures in the vicinity of the trapped ion cell of $10^{-6}$ torr were found conducive to efficient ion trapping. Unfortunately, the high neutral density in the trapped ion cell also caused rapid dephasing of the excited ion cloud, resulting in short-lived transients and low-resolution measurements. Studies in which ions were injected into the cell at $10^{-10}$ torr were unsuccessful owing to the low collision frequency at that pressure and concomitantly low accumulated trapping efficiency. Thus, the conditions for optimum ion accumulation and optimum in detertion are mutually exclusive unless separated in time. The combination of the magnetically compatible pulsed valve and the custom cryopumping assembly described earlier allows both high pressure, for enhanced ion accumulation and subsequent cooling, and rapid pumpdown to low pressure (i.e., $\leq 10^{-9}$ torr) for excitation and detection. During ion injection, the pressure in the cell rises to approximately $10^{-4}$ torr, providing both effective ion accumulation over a wide range of ion kinetic energies and rapid $z$ axis cooling. Preliminary data indicate that heavier ions are discriminated against when using accumulated trapping in these pressure regimes. I ligh signal-to-noise spectra have been obtained for species as large as bovine serum albumin $\left(M_{\mathrm{r}} 66 \mathrm{kDa}\right)$, but very long valve pulses are required. Attempts at trapping larger ions have been for the most part unsuccessful. The high-speed pumping in the analyzer region typically lowers the cell pressure to below $10^{\circ}$ torr in $5 \mathrm{~s}$ (or less, depending on the width of the gas pulse). Thus, the combination of these components creates nearly ideal pressure conditions for ion trapping, cooling, and detection.

\section{Excitation and Detection}

Once an adequate number of ions is confined in the trapped ion cell, there are a variety of parameters that can have a profound influence on the obtainable FT-ICR signal. If the ion cloud has not sufficiently cooled to the center of the trapping well, or if an inappropriate excitation is applied, parameters other than pressure may prove to be the factors that limit transient lifetime. Below is a brief description of several parameters that have proved to be critical in obtaining highperformance ESI/FT-ICR spectra. 


\section{Trap Potentials During Excitation and Detection}

As detailed elsewhere [26], the potentials at which ions are most effectively accumulated and most effectively detected are often quite dissimilar. We have observed that, up to the point where ion loss occurs, lower trapping potentials used during excitation and detection result in longer transient lifetimes. Ions are typically accumulated and cooled at potertials that muimic the kinetic energy (divided by ion charge) of the injected ion beam, typically $0.5-10 \mathrm{~V}$. Prior to RF excitation, the accumulation potential is lowered nonadiabatically to the detection potential, which is typically $150-250 \mathrm{mV}$. The minimum magnitude of the detection trap potential is highly dependent on cell cleanliness because charging on either trap plate can significantly perturb the shallow electrostatic well. When ion accumulation occurs at several volts, transient duration can be improved by more than an order of magnitude if the trapping potential is lowered to several hundred millivolts prior to excitation and detection. This phenomenon is most likely due to the increased radial electric field experienced by the ion cloud when larger trapping potentials are used. This undesirable effect might be further minimized with the use of an elongated (IonSpec Corp., Irvine, CA) [34] or screened [35] trapped ion cell, both of which have inherently lower radial electric fields.

\section{Ion Cooling}

Although the cubic trapped ion cell $[36,37]$ is one of the most widely utilized geometries for FT-ICR, there are several inherent limitations owing to the nonideal electric tields, which must be considered to achieve the highest possible performance. The potential along the $z$-axis is approximately harmonic, with the degree of anharmonicity increasing with proximity to the trapping electrodes. It is desirable, from a trapping standpoint, for the ion cloud to reside in the center of the potential well, where the trapping field is most nearly ideal. Additionally, because the excitation electrodes terminate at the cell boundary, the electric field due to RF excitation is not constant over the length of the cell. Thus, from an excitation/detection standpoint, it is also desirable to have an ion packet that has relaxed to the center of the cell, where the axial component of the excitation field is less significant and where the amplitude of the excitation is more nearly uniform. As described above, a pulse of $\mathrm{N}_{2}$ gas is admitted to the cell region, coinciding with the ion injection event. This short-lived pressure increase, in addition to enhancing the accumulated trapping process, greatly enhances the rate of $z$-axis ion relaxation because the ion-neutral collision frequency is greatly increased. Following the pulsed gas introduction, the trapping potentials are maintained at the accumulation potential for 1-60 s to allow the ion population to cool along the $z$-axis. Preliminary studies indicate that the neces- sary cooling delay is dependent on both the mass of the ion and the duration of the gas pulse. For examf le, bovine insulin demonstrates a near-linear increase in transient half-life for delays up to approximately $45 \mathrm{~s}$, after which longer delays do not decrease the rate of transient decay. For much lighter species, such as a polypeptide mixture from a tryptic digest of $\mathrm{cy}$ tochrome $c$ having a mass range $150-2400 \mathrm{Da}$, cooling appears to take place at a higher rate. These lighter species are particularly sensitive to background pressure, and the cell must be pumped down to base pressure before long-lived transients can be obtained.

\section{Excitation Voltage}

All spectra presented here were acquired following swept excitation over the corresponding detection bandwidth. The sweep rate of this chirp excitation was typically $50 \mathrm{~Hz} / \mu \mathrm{s}$ for the broadband mode and 25 $\mathrm{Hz} / \mu \mathrm{s}$ for the narrow-band mode. In all cases, regardless of bandwidth, the amplitude of the applied chirp was found to affect both transient intensity and duration. For example, the transient for bovine insulin shown in Figure 3 was acquired following a $25-\mathrm{Hz} / \mu \mathrm{s}$ chirp excitation from 65,566 to $77,266 \mathrm{~Hz}$ at $70 \mathrm{~V}_{\mathrm{pop}}$. The observed transient lifetime is in excess of $80 \mathrm{~s}$. If a $74-V_{\mathrm{p}-\mathrm{p}}$ chirp is applied under identical conditions, the initial amplitude of the transient is higher, but a transient lifetime of less than $40 \mathrm{~s}$ is observed. Thus, to obtain ultrahigh resolution for large biomolecules, fine control over the excitation amplitude is essential.

\section{Broadband Detection and High-Resolution ESI $/ F T$-ICR}

Broadband mass spectra were obtained for bovine insulin $\left(M_{\mathrm{r}} 5734\right)$, bovine ubiquitin $\left(M_{\mathrm{r}} 8565\right)$, bovine cytochrome $c\left(M_{\mathrm{r}} 12,231\right)$, RNase $\mathrm{A}\left(M_{\mathrm{r}} 13,682\right)$, RNase B $\left(M_{\mathrm{r}} 14,899\right)$, and equine myoglobin $\left(M_{\mathrm{r}} 16,951\right)$. Figure 4 shows a typical ESI mass spectrum for equine myoglobin sprayed from a $5 \%$ acetic acid solution obtained with the trap plate potentials at $8 \mathrm{~V}$ for ion accumulation. This spectrum was acquired in the broadband mode at an $\mathrm{ADC}$ rate of $200 \mathrm{kHz}$, with $256 \mathrm{~K}$ data points collected.

A characteristic of all the ESI mass spectra initially obtained was that the charge state distributions were shifted somewhat toward higher mass-to-charge ratios (lower charge state) than those obtained on conventional quadrupole mass spectrometers. The observed ESI distribution appears to be the result of complex solution, ESI droplet, and gas-phase processes [2]; the initial charge state distribution can be modified by subsequent deprotonation reactions in the gas phase and by Coulombic contributions to each of these reactions [38, 39]. Additionally, the long, heated capillary may also contribute to the observed shift by increasing 


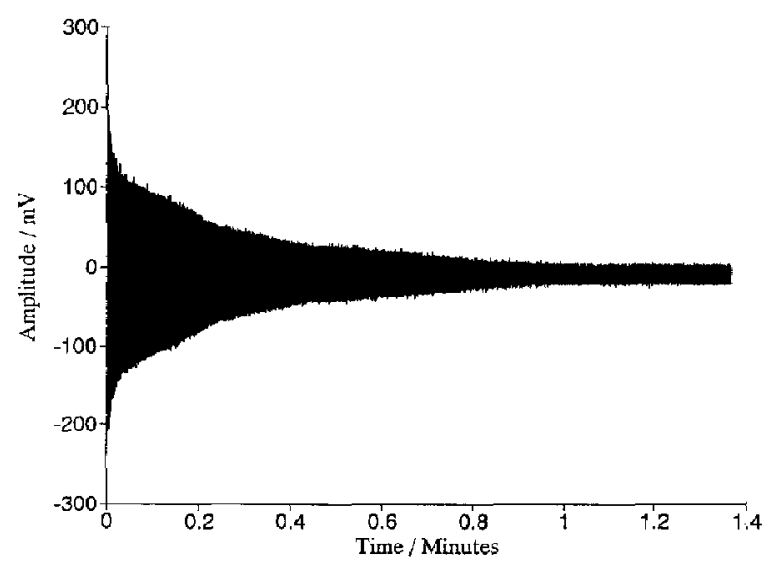

Figure 3. A greater than 80 -s bovine insulin transient recorded at approximately $10^{-y}$ torr using the alternate data acquisition system described in the text.

the proton transfer probability. Alternatively, other results indicate that trapping conditions can affect the charge state distribution observed [26]. The charge state distributions observed at low trapping voltages in the present work are still shifted to high mass-tocharge ratios compared with quadrupole results, which suggests that both factors may contribute.
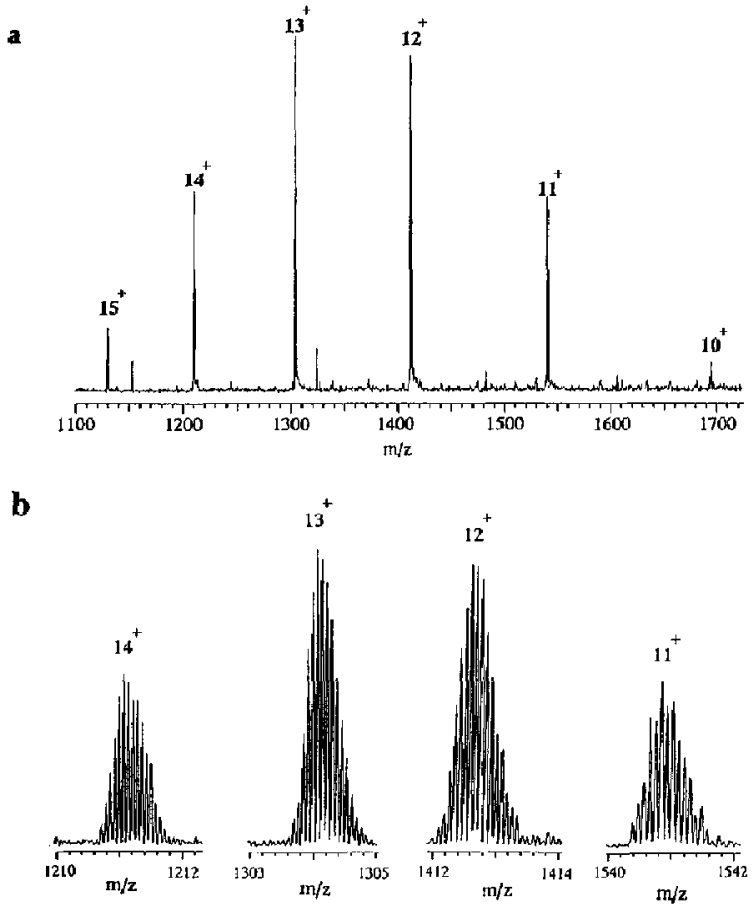

Figure 4. (a) Full (broadband) positive ion ESI mass spectrum obtained for equine myoglobin in a $5 \%$ acetic acid solution; (b) expansion of the mass scale about several of the charge states showing resolution of the isotopic envelopes.
Figure $4 \mathbf{b}$ shows the resolved isotopic envelope obtained for several charge states of horse myoglobin. The average mass resolution $(m / \Delta m)$ obtained for the $13+$ charge state was 58,000 , with a sine-bell apodization applied to the time-domain signal. The average mass resolution for the unapodized spectrum was 103,000 . This resolution is more than sufficient to determine the actual charge state from the spacing of peaks in the isotopic envelope of each ion, and the signal intensities and signal to noise are high enough that the isotopic distribution closely matched the theoretically predicted distribution. From the theoretical isotope distribution for equine myoglobin, the most abundant ion contains $11{ }^{13} \mathrm{C}$ (or equivalent) isotopic contributions. Using this ion from each isotopic distribution, we calculated the molecular weight to be $16,950.84 \pm 0.04$. whereas the theoretical molecular weight for the isotope ion containing $11{ }^{13} \mathrm{C}$ isotopes is 16,951.02. This corresponds to a mass measurement error of $10 \mathrm{ppm}$. In this example, the relative abundance of the monoisotopic molecular ion is substantially less than $1 \%$. To determine accurately the molecular weight from the isotopic distribution, an accurate assignment of each isotope peak (i.e., the number of ${ }^{13} \mathrm{C}$ isotopes contained in the ion) is needed. As the signal intensity decreases, the relative abundances in the observed isotopic distribution become less well defined, making it increasingly difficult to assign accurately the extent of ${ }^{13} \mathrm{C}$ contribution to each peak. Therefore, high resolution in itself may not always be sufficient to obtain highly accurate molecular weight measurements. Signal intensities high enough to obtain ion statistics sufficient to define each isotopic distribution are also required.

The error that may result from low ion intensities is a major concern when deciding on a calibrant to use to obtain accurate mass measurements. For accurate mass analysis, it appears that the following criteria facilitate obtaining an accurate calibration. First, a protein used as a calibrant should contain a sufficient number of charge states to span the mass-to-charge ratio range of interest. More important, however, the monoisotopic contribution to the isotopic distribution of the protein should be sufficient to allow unambiguous assignment of this peak for each charge state. Even though the monoisotopic ion is not abundant for moderate-sized proteins, accurate assignment of this ion for the calibrant is vital to correct assignments of the more abundant ions. Without this, the probability of choosing the incorrect ion in the isotope distribution can be quite high. The protein chosen as a calibrant in the present studies was bovine ubiquitin, whose charge state distribution spans from $m / z 1071\left(8^{+}\right)$to $m / z 2142\left(4^{+}\right)$ and whose monoisotopic ion relative intensity is approximately $4 \%$.

"Real-world" biopolymers generally present greater challenges because they are almost always mixtures. Ribonucleases have been widely studied as a model for understanding protein folding and determining 
protein structure-function relationships [40]. The predominant form is RNase A, which is a protein containing 124 amino acid residues and four disulfide bridges. RNase $B$ is a glycoprotein in which the major form is known to consist of RNase $A$ and an oligosaccharide [41] that consists of five mannose and two $N$ acetylglucosamine moieties, $\mathrm{Man}_{5} \mathrm{GlcNac}_{2}$, with additional variations in the carbohydrate moiety that give rise to considerable microheterogeneity. ESI of RNase $B$ has been shown to yield interpretable mass spectra [42].

Figure 5a shows the positive-ion ESI mass spectrum of RNase B from a $5 \%$ aqueous acetic acid solution obtained with $37 \mathrm{~W}$ applied to the inlet capillary (approximately $100{ }^{\circ} \mathrm{C}$ ); Figure $5 \mathrm{~b}$ shows an expanded view of the $8^{+}$charge state of the molecular ion region. Along with the molecular ion, which was a relatively minor constituent, peaks were observed corresponding to one, three, and four additional hexose moieties (assumed to be mannose additions on the basis of previous results [42]). In addition, the nonspecific association of several neutral $\mathrm{H}_{3} \mathrm{PO}_{4}$ molecules with both the molecular in species and the higher

a

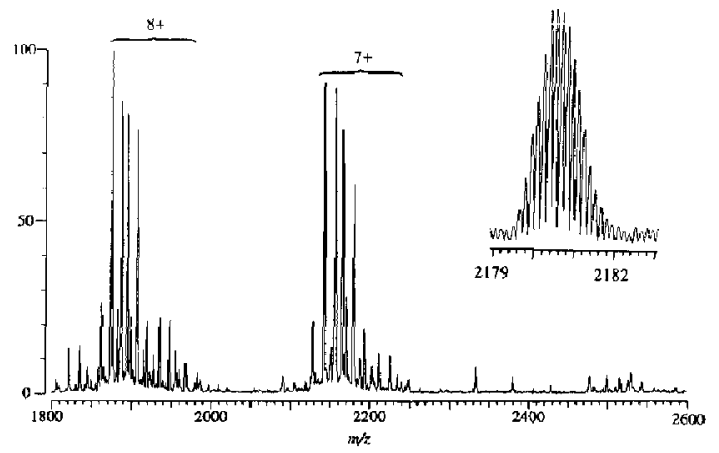

b

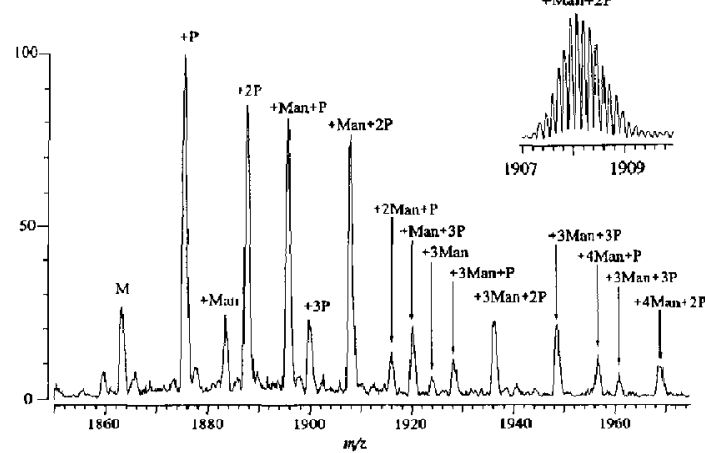

Figure 5. (a) Positive ion ESI mass spectrum (with $37 \mathrm{~W}$ applied to the inlet capillary) of RNase B from a $5 \%$ acetic acid solution; (b) expanded view of the $8^{+}$charge state region of RNase $B$ showing peaks resulting from multiple additions and adductions of mannose (Man) and $\mathrm{H}_{3} \mathrm{PO}_{4}(\mathrm{P})$, respectively; $\mathrm{M}$, RNase $B$ molecular ion; insets, single peaks demonstrating resolution sufficient to resolve the isotopic envelope. glycosylated forms was observed. As shown by the inset in Figure 5, sufficient resolution was obtained to easily resolve the isotopic envelopes for all components, which allowed the determination of the charge state of each ion. This, in turn, allowed for an accurate molecular weight determination of each species present in this sample. The calculated molecular weight of the RNase B molecular ion from the $8{ }^{13} \mathrm{C}$ isotope peak was $14,898.74 \pm 0.05$, compared with a theoretical molecular weight of $14,898.71$, corresponding to an error of approximately 2 ppm. Table 1 compares the theoretical and experimental molecular weights for RNase B and its higher glycosylated and phosphatecontaining analogs. The relative crror ranged from less than 1.0 to $4.5 \mathrm{ppm}$ for the species, with up to four additional moieties, demonstrating the potential for obtaining high-resolution precision mass measurements of complex biopolymer mixtures.

Thermally induced dissociation in a heated capillary [43] has proved to be an effective method for dissociating multiply charged ions produced by ESI. Figure 6 illustrates the use of increased thermal energy to eliminate the noncovalently assoniated phosphate from RNase $B$ and its higher glycosylated analogs. Figure $6 a$ shows the entire broadband mass spectrum of RNase B with $52 \mathrm{~W}$ applied to the inlet capillary, and Figure $6 \mathrm{~b}$ is an expanded view of the $8^{+}$molecular ion region. With increased capillary heating, the only species observed in Figure $6 \mathrm{~b}$ are the glycoprotein molecular ion and the higher glycosylated forms. The molecular weights determined for RNase B and for the species with onc, two, and three additional mannose residues from this spectrum correlate quite well with the data shown in Figure 5.

\section{High Sensitivity}

The high-sensitivity capabilities have the potential of making FT-ICR coupled with ESI into a powerful tool for analytical separations. Discrepancies still exist, however, with respect to the time needed to acquire a high-resolution FT-ICR mass spectrum and the width of a typical chromatographic peak or the higher resolved peaks of capillary electrophoresis [44]. To illustrate the high sensitivity of the present FT-ICR system, $4.3 \mathrm{fmol}$ of bovine insulin and $3.9 \mathrm{fmol}$ of bovine ubiquitin, respectively, were consumed to acquire the mass spectra shown in Figure 7 . The experimental conditions included a sample flow rate of $100 \mathrm{~nL} / \mathrm{min}$ for an approximately $10^{-4} \mathrm{M}$ solution and an ion injection time of $25 \mathrm{~ms}$. Acquired in the heterodyne mode with a $50-\mathrm{kHz}$ ADC rate, the resolution of the $4^{+}$ charge state of insulin (Figure 7a) was 180,000, much more than sufficient to resolve the isotopic envelopes and to allow determination of the charge states. In addition to the molecular ion, the spectrum for ubiquitin shows smaller contributions due to sodium adduction, loss of water from the molecular ion, and a peak $114.01 \mathrm{u}$ less than the molecular ion. Previous studies 
Table 1. Comparison of theoretical and experimentally determined molecular weights for the most intense peak in the isotopic envelope ${ }^{a}$ for RNase $B$, higher glycosylated analogs, and RNase $\mathrm{B} / \mathrm{H}_{3} \mathrm{PO}_{4}$ adducts

\begin{tabular}{lccc}
\hline & Theoretical & Experimental & $\begin{array}{c}\text { Error } \\
\text { (ppm) }\end{array}$ \\
\hline \hline RNase B & $14,898.71$ & $14,898.74$ & 2.0 \\
$+\mathrm{H}_{3} \mathrm{PO}_{4}$ & $14,996.69$ & $14,996.72$ & 2.0 \\
$+1 \mathrm{Mannose}^{2}$ & $15,060.76$ & $15,060.79$ & 2.0 \\
$+2 \mathrm{H}_{3} \mathrm{PO}_{4}$ & $15,094.67$ & $15,904.67$ & $<1.0$ \\
$+1 \mathrm{Mannose}^{-1} \mathrm{H}_{3} \mathrm{PO}_{4}$ & $15,158.74$ & $15,158.76$ & 1.3 \\
$+3 \mathrm{H}_{3} \mathrm{PO}_{4}$ & $15,192.65$ & $15,192.65$ & $<1.0$ \\
+2 Mannose & $15,222.82$ & $15,222.87$ & 3.3 \\
+1 Mannose $+2 \mathrm{H}_{3} \mathrm{PO}_{4}$ & $15,256.72$ & $15,256.73$ & $<1.0$ \\
+2 Mannose $+1 \mathrm{H}_{2} \mathrm{PO}_{4}$ & $15,320.80$ & $15,320.83$ & 2.0 \\
+1 Mannose $+3 \mathrm{H}_{3} \mathrm{PO}_{4}$ & $15,354.70$ & $15,354.67$ & -2.0 \\
+3 Mannose & $15,384.87$ & $15,384.87$ & $<1.0$ \\
+2 Mannose $+2 \mathrm{H}_{3} \mathrm{PO}_{4}$ & $15,418.78$ & $15,418.78$ & 2.6 \\
+3 Mannose $+1 \mathrm{H}_{3} \mathrm{PO}_{4}$ & $15,482.85$ & $15,482.89$ & \\
+2 Mannose $+3 \mathrm{H}_{3} \mathrm{PO}_{4}$ & $15,516.76$ & Not observed & 1.5 \\
+4 Mannose & $15,546.92$ & $15,546.99^{\circ}$ & 2.6 \\
+3 Mannose $+2 \mathrm{H}_{3} \mathrm{PO}_{4}$ & $15,580.83$ & $15,580.87$ & 3.2 \\
+4 Mannose $+1 \mathrm{H}_{3} \mathrm{PO}_{4}$ & $15,644.90$ & $15,644.95$ & 1.9 \\
+3 Mannose $+3 \mathrm{H}_{3} \mathrm{PO}_{4}$ & $15,678.81$ & $15,678.79$ & 1.9 \\
+4 Mannose $+2 \mathrm{H}_{3} \mathrm{PO}_{4}$ & $15,742.88$ & $15,742.91$ & \\
\hline
\end{tabular}

${ }^{a}$ The peak $9 \mathrm{Da}$ higher than the monoisotopic species and corresponding to $8{ }^{13} \mathrm{C}$ (or equivalent) isatope substitutions.

${ }^{b}$ Obtained from the higher capillary temperature experiment illustrated in Figure 6.

indicate that this species is most likely due to an in vitro proteolytic artifact, resulting in the elimination of two glycine residues at the carboxy terminus $[45,46]$, which results in a loss of $114.04 \mathrm{u}$. These results indicate the feasibility of obtaining useful mass spectra while consuming only a small amount of material. The actual implementation of high-sensitivity ESI/FT-ICR for "real-world" samples will be most effectively realized in conjunction with on-line microcolumn separation methods (i.e., capillary HPLC or capillary electrophoresis). Recently, this laboratory has demonstrated improved capillary electrophoresis/quadrupole mass spectrometry methods that have allowed mass spectra to be obtained with only 600 amol of protein [47]. The transfer of this capability to FT-ICR, while not straightforward, has the promise of creating new opportunities for biochemical research.

\section{Ultrahigh-Resolution ESI/FT-ICR}

The mass resolution required to determine accurately the charge state of the proteins studied (i.e., observe the ${ }^{13} \mathrm{C}$, or equivalent, isotope distribution for each charge state) is typically comparable to the molecular weight of the protein under investigation. For the present studies, this corresponds to a resolution of no more than 25,000 . Ultrahigh resolution becomes increasingly important when trying to solve real biologi- cal problems, such as separation of protein variants or adducts of very similar mass. For example, mass resolution in excess of 500,000 is required to resolve (with a $50 \%$ valley definition) equimolar quantities of a single-chain hemoglobin mudified by addition of lluree methyl groups versus addition of one acetyl group. The required resolution increases by one to two orders of magnitude to separate components of the isotopic fine structure owing to the different mass defects of isotopic contributions. Amino acid substitutions in large proteins can result in small mass defects [48] that require increasingly higher mass resolution so as to distinguish certain variants (e.g., $0.036 \mathrm{Da}$ for a lysine/glutamic acid substitution). Many other examples exist in which multiple adductions and the inherent heterogeneity of many biopolymers complicate the mass spectrum and require higher mass resolution.

To obtain ultrahigh resolution for larger molecules, longer time-domain signals are required. This requirement demands not only the environment to produce long transients (i.e., low pressures and nearly ideal electric and magnetic fields in the trapping region), but also the ability to collect and transform quite large data sets. Due to memory restrictions, the present IonSpec Omega data system was unable to record (in the heterodyne mode with an ADC rate of $50 \mathrm{kHz}$ ) the full time-domain signal for several electrosprayed protein ions, once cell potentials and excitation conditions had been optimized. Although it is true that the rate of 


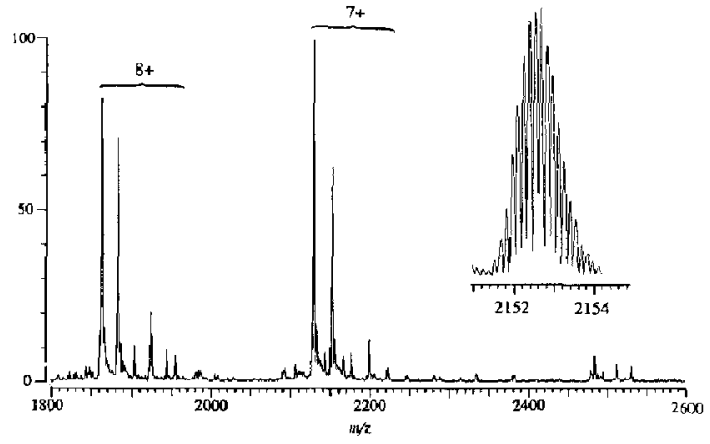

b

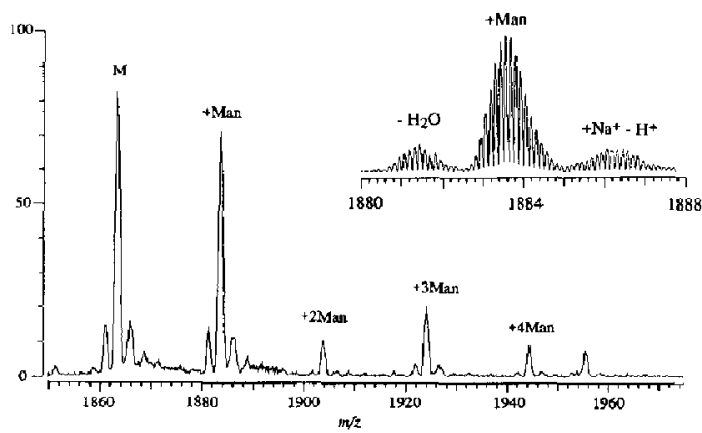

Figure 6. (a) Positive ion ESI mass spectrum (with $52 \mathrm{~W}$ applied to the inlet capillary) of RNase B from a $5 \%$ acetic acid solution; (b) expanded view of RNase B ( $8^{+}$charge state region) showing peaks resulting from multiple adduction/addition and the decrease in $\mathrm{H}_{3} \mathrm{PO}_{4}$ addurtion observed with greater capillary heating. Man, mannose; $M$, RNase $B$ molecular ion; insets, single peaks demonstrating resolution sufficient to resolve the isotopic envelope.

the ADC could be reduced (digitization at $3 \mathrm{kHz}$ would allow the full observation of an 80-second time-domain signal with the standard $256 \mathrm{~K}$ memory allocation of the Ionspec data station), other complications arise during operation at low ADC rates. For example the commercial system requires the selection of the same bandwidth for both excitation and detection. This can be a distinct advantage in some cases, preventing the observation of some ions whose frequencies occur outside the bandwidth but, when excited, are aliased or observed as "reflected" peaks [49]. In the present situation, however, the simultaneous selection of the two bandwidths increases the difficulty associated with the determination of proper excitation conditions. As such, long transients could not be acquired with the acquisition bandwidth set below 50 $\mathrm{kHz}$. The alternative data acquisition scheme described earlier (see Experimental) was developed to allow the acquisition of very long transient lifetimes.

Shown in Figure 3 is the time-domain signal obtained in the heterodyne mode for insulin in the $4^{+}$ charge state $(\mathrm{m} / \mathrm{z} 1434)$. Transformation of only the last $5 \mathrm{~s}$ of the transient illustrated in Figure 3 produced

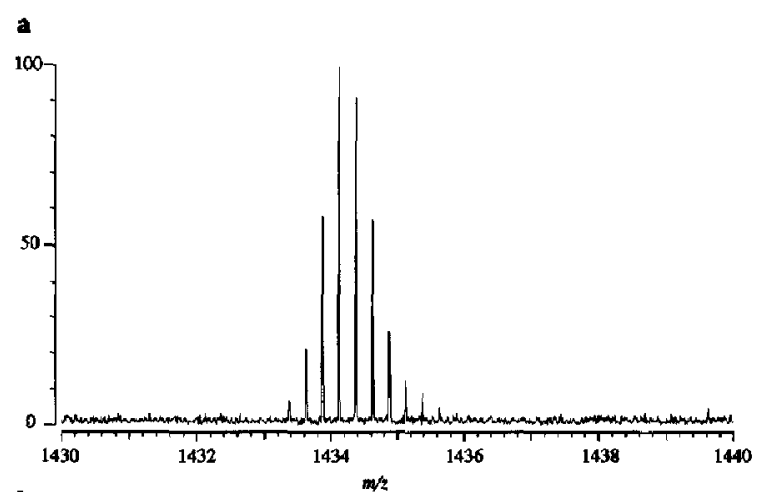

b

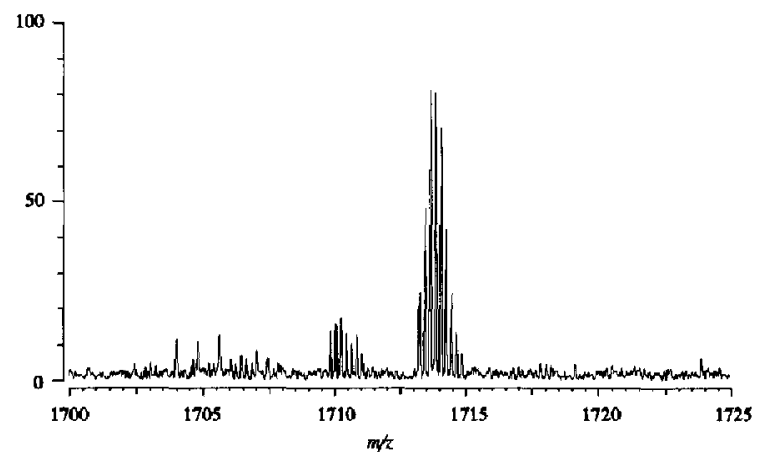

Figure 7. (a) Positive ion ESI mass spectrum of bovine insulin acquired with the consumption of $4.3 \mathrm{fmol}$ of the protein. Shown is the $4^{+}$charge state region. (b) Positive ion ESI mass spectrum of bovine ubiquitin acquired with the consumption of $3.9 \mathrm{fmol}$ of the protein. Shown is the $5^{+}$charge state region. A smaller distribution corresponding to water loss from the molecular ion is also evident.

isotopically resolved peaks for the $4^{+}$charge state of insulin, the largest of which was roughly five times the noise level, indicating that the observable signal did exist at least $80 \mathrm{~s}$ after the excitation pulse. The highest resolution mass spectrum ( 700,000 full-width at halfmaximum) obtained from this transient was produced by transforming only the last $40 \mathrm{~s}$ of data, and is illustrated in Figure 8. This resolution is less than is theoretically attainable from $4 \mathrm{U}$ s of data, and, in fact, transformation of the entire data set produces mass resolution significantly less than that shown in Figure 8. This resolution limitation is due to an observed shift toward higher cyclotron frequency as the cyclotron radii become smaller. The cause of this shift is currently under investigation and will be discussed in detail in a future publication. These results demonstrate that transients of exceptional length can be obtained from multiply charged proteins by ESI/FT-ICR, and we are optimistic that much higher resolution can be achieved than demonstrated to date. Most important, however, is that for proteins up to approximately $20 \mathrm{kDa}$, resolutions of $10^{5}$ are routinely achieved, providing many important opportunities for biopolymer characterization. 


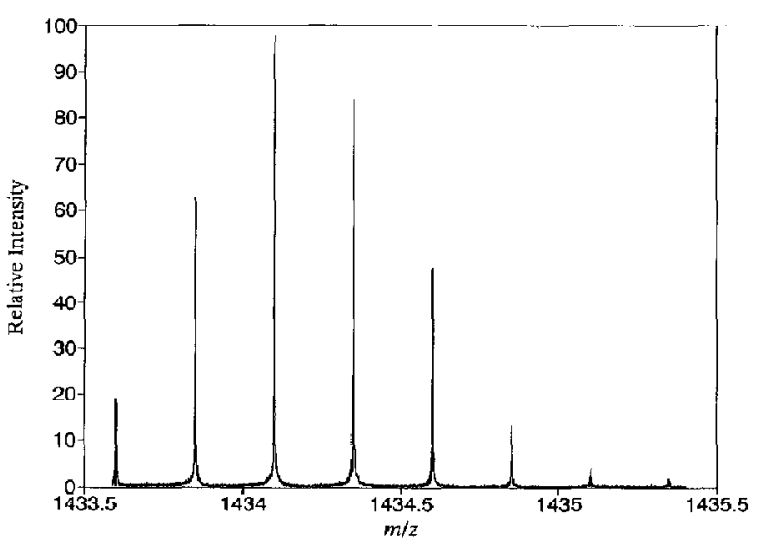

Figure 8. High-resolution $(>700,000)$ positive ion ESI mass spectrum of bovine insulin ( $4^{+}$charge state) obtained by Fourier transformation of the last $40 \mathrm{~s}$ of data from the transient shown in Figure 3.

\section{Conclusions}

This report describes our initial ESI/FT-ICR results obtained for small proteins and represents progress over less than four months since initial instrument operation. Thus, we view both the resolution and sensitivity results demonstrated thus far as only preliminary and anticipate that further investigations along the lines suggested in this report will lead to further significant improvements. Nevertheless, we believe that the capabilities demonstrate that the ESI combination with FT-ICR has the potential of grcatly facilitating biochemical research. The long transient lifetimes obtained in this report highlight the need for advanced data acquisition and manipulation techniques and suggest that the extension to large biopolymers will depend on better understanding and control of ion cooling, as well as obtaining more ideal ion trapping and excitation conditions.

\section{Acknowledgments}

This work was supported by the Molecular Science Research Center of Pacific Northwest Laboratory and the Director, Office of Health and Environmental Research, US Department of Energy. Pacific Northwest Laboratory is operated by Battelle Memorial Institute for the US Department of Energy under Contract DE-AC06-76RLO 1830.

\section{References}

1. Dole, M.; Mack, L. L.; Hines, R. L.; Mobley, R. C.; Ferguson, L. D.; Alice, M. B. I. Chem. Phys. 1968, 49, 2240-2249.

2. Yamashita, M.; Fenn, J. B. J. Phys. Chem. 1984, 88, 4451.

3. Aleksandrov, M. L.; Gall, L. N.; Krasnov, V. N.; Nikolaev, V. I.; Pavlenko, V.A.; Shkurov, V.A. Dokl. akad. Nauk SSSR 1984, 277, 379.

4. Fenn, J. B.; Mann, M.; Meng, C. K.; Wong, S. F.; Whitehouse, C. M. Mass Spectrom. Rev. 1990, 9, 37.

5. Smith, R. D.; Loo, J. A.; Ogorzalek Loo, R. R.; Busman, M.; Udseth, H, R. Mass Spectrom, Rev. 1991, 10, 359.
6. Meng, C. K.; McEwen, C. N.; Larsen, B. S. Rapid Commun Mass Spectrom. 1990, 4, 147.

7. (a) Dodenov, A. F.; Chernushevich, I. V.; Laiko, V. V. Presented at the 12th International Mass Spectrometry Conference, Amsterdam, The Netherlands, August 26-30, 1991; (b) Boyle, J. G.; Whitehouse, C. M.; Fenn, J. B. Rapid Commun. Mass Spectrom. 1991, 5, 400; (c) Boyle, J. G.; Whitehouse, C. M. Anal. Chem. 1992, 64, 2084.

8. (a) Van Berkel, G. J.; Glish, G. L.; McLuckey, S. A. Anal. Chem. 1990, 62, 1284; (b) McLuckey, S. A.; Van Berkel, G. J.; Glish, G. L.; Huang, E. C.; Henion, J. D. Anal. Chem. 1991, 63, 375; (c) Schwartz, J. C.; Syka, J. E. P.; Jardine, 1. Proceedings of the 39th ASMS Conference on Mnss Spectrametry and Allied Topics; Nashville, TN, May 19-24, 1991; p 540.

9. (a) Henry, K. D.; Williams, E. R.; Wang, B. H.; McLafferty, F. W.; Shabanowitz, J.; Hunt, D. W. Proc. Natl. Acad. Sci. USA 1989, 86, 975-978; (b) Henry, K. D.; McLafferty, F. W. Org. Mass. Spectrom. 1990, 25, 490-492; (c) Henry, K. D.; Quinn, J. P.; McLafferty, F. W. J. Am. Chem. Soc. 1991, 113, 5447; (d) Beu, S. C; Senko, M. W.; Quinn, J. P.; McLafferty, F. W. I. Am. Sac. Mass Spectrom. 1993, 4, 190.

10. (a) Loo, J. A.; Ogorzalek Loo, R. R.; Udseth, H. R.; Edmonds, C. G.; Smith, R. D. Rapid Commun. Mass Spectrom. 1991, 5, 101; (b) Brown, C.; Camilleri, P.; Haskins, N. J.; Sanders, M. J. Chem. Soc. Chem. Commun. 1992, 761; (c) Viswanatham, K; Chait, B. T. Rapid Conmun. Mass Spectrom. 1991, 5, 214; (d) Gvevremont, R.; Siu, K. W. M.; Le Blanc, J. C. Y.; Berman, S. S. J. Am. Soc. Mass Spectrom. 1992, 3, 216.

11. Barinaga, C. J.; Edmonds, C. G.; Udseth H. R.; Smith, R. D. Rupid Comtrnur. Mass Spectrom. 1989, 3, 160-164.

12. Loo, J. A.; Edmonds, C. G.; Smith, R. D. Anal. Chem. 1991, 63, 2488-2499.

13. (a) Mann, M.; Meng, C. K.; Fenn, J. B. Anal. Chem. 1989, 61. 1702; (b) Reinhold, B. B.; Reinhold, V. N. J. Am. Soc. Mass. Spectrom. 1992, 3, 207-215.

14. Cody, R. B; Tamura, J.; Musselman, B. D. Presented at the 4th International Symposium on Tandem Mass Spectrometry, Lake Louise, Alberta, Canada, November 20-24, 1991.

15. Marshall, A. G.; Grosshans, P. B. Anal. Chem. 1991, 4, 215 A.

16. Gulcicek, E. E.; Whitehouse, C. M. Proceedings of the 40 th ASMS Conference on Mass Spectrometry and Allied Topics; Washington, DC, May 31-June 5, 1992; p 1649.

17. Lebrilla, C. B.; Amster, I. J.; McIver, R. T. Jr. Int. I. Mass Spectrom. Ion Processes 1989, 87, R7-R13.

18. Maruyama, S.; Anderson, L. R.; Smalley, R. E. Rev, Sci. Instrum. 1990, 61, 3686-3693.

19. Smith, R. D.; Loo, J. A.; Barinaga, C. J.; Udseth, H. R. Anal. Chem. 1988, 60, 1948-1952.

20. Katta, V.; Chait, B. T. Rapid Commun. Mass Spectrom. 1991, 5, 214.

21. Loo, J. A.; Udseth, H. R.; Smith, R. D. Rapid Commun. Mass Spectrom. 1988, 2, 207-210.

22. Hofstadler, S. A.; Beu, S. C.; Laude, D. A. Anal. Chem. 1993, 65, 312-316.

23. Feng, R.; Konishi, Y. Proceedings of the 39th ASMS Conference on Mass Spectrometry and. Allied Topics, Nashville, TN, May 19-24, 1991; pp 260-261.

24. Hofstadler, S. A.; Laude, D. A. Jr. Int. J. Mass Spectrom. Ion Processes 1990, 101, 65-78.

2b. Hofstadler, S. A,; Laude, D. A. Jr. Anal. Chem. 1992, 64, $569-572$.

26. Hofstadler, S. A.; Laude, D. A. Jr. J. Am. Soc. Mass Spectrom. 1992, 3, 615 .

27. Caravatti, P. US Patent $4,924,089$.

28. Mclver, $R$. T. US Patent 4,535,235.

29. Sharp, T. E.; Eyler, J. R.; Li, E. Int. J. Mass Spectrom. Ion Processes 1972, 9, 421-439. 
30. Huang, S. K.; Rempel, S. K.; Gross, M. L. Int. I, Mass Spectrom. Ion Processes 1986, 72, 15-31.

31. Byrne, J.; Farago, P. S. Proc. Phys. Soc. 1965, 86, 801-820.

32. Savard, G.; Becker, S.; Bollen, G.; Kluge, H.-J.; Schweikhard, L.; Stolzenberg, H.; Wiess, U. Phys. Lett. 1991, 158, 247-252.

33. Bollen, G.; Moore, R. B.; Saverd, G.; Stolzenberg, H. Appl. Phys. 1990, 68, 4355 .

34. Hunter, R. L; Sherman, M. G.; McIver, R. T. Jr. Int. J. Mass Spectrom. Ion Processes Ion Phys. 1983, 50, 259-274.

35. Wang, M.; Marshall, A. G. Anal. Chemt. 1989, 61, 1288-1293.

36. Comisarow, M. B. Adv. Mass Spectrom. 1980, 8, 1698-1706.

37. Comisarow, M. B. Int. J. Mass Spectrom. Ion Processes 1981, 37, 251-257.

38. (a) Chait, B. T.; Chowdhury, S. K.; Katta, V. Proceedings of the $39 \mathrm{th}$ Conference on Mass Spectrometry and Allied Topics; Nashville, TN, May 19-24, 1991; p 447; (b) Schweikhard, L.; Guaiy, S.; Marshall, A. G. Int. I. Mass Spectrom. Ion Processes $1992,120,71$.

39. Winger, B. E.; Light-Wahl, K. J; Smith, R. D. J. Am. Soc. Mass Spectrom. 1992, 3, 624 .
40. Voet, D.; Voet, J. G. Biochemistry; Wiley: New York, 1990; pp 260-269.

11. Scott, T.; Eagleson, M., Eds. Concise Encyclopedia of Biochem istry, 2nd ed., Walter de Gruyter: Berlin, 1988; p 531.

42. Gillece-Castro, B. L; Bourell, J. H.; Stults, J. T. Proceedings of the 39th ASMS Conference on Mass Spectrometry and Allied Topics, Nashville, TN; May 19-24, 1991; p 276.

43. Rockwood, A. L.; Busman, M.; Smith, R. D. Rapid Commun. Mass. Spectrom 1991, 5, 582.

44. Olivares, J. A.; Nguyen, N. T.; Yonker, C. R.; Smith, R. D. Anal. Chem, 1987, 59, 1230-1232.

45. Vijay-Kuman, S.; Bugg, C. G.; Cook, W. J. J. Mol. Biol. 1987, $194,531$.

46. Wilkinson, K. D.; Audhya, T. K. T. Biol. Chem. 1981, 256, 9235.

47. Wahl, J. H.; Goodlett, D. R.; Udseth, H. R.; Smith, R. D. Anal. Chem. 1992, 64, 3194-3196.

48. Wada, Y. Biol. Mass Spectrom. 1992, 21, 617.

49. Cody, R. B.; Kinsinger, J. A.; Goodman, S. D. Anal. Chem. 1987, 59, 2567. 